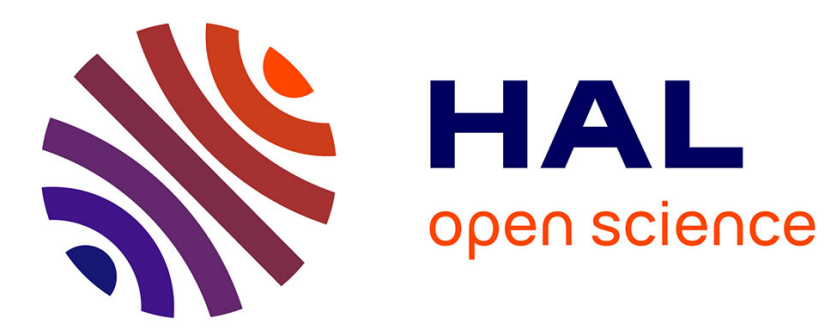

\title{
Vibration-induced compaction of granular suspensions
}

S. Kiesgen de Richter, Caroline Hanotin, Philippe Marchal, Sébastien Leclerc, Franck Demeurie, N. Louvet

\section{To cite this version:}

S. Kiesgen de Richter, Caroline Hanotin, Philippe Marchal, Sébastien Leclerc, Franck Demeurie, et al.. Vibration-induced compaction of granular suspensions. European Physical Journal E: Soft matter and biological physics, 2015, 38 (7), 9p. 10.1140/epje/i2015-15074-7 . hal-01266900

\section{HAL Id: hal-01266900 \\ https://hal.univ-lorraine.fr/hal-01266900}

Submitted on 24 Jan 2022

HAL is a multi-disciplinary open access archive for the deposit and dissemination of scientific research documents, whether they are published or not. The documents may come from teaching and research institutions in France or abroad, or from public or private research centers.
L'archive ouverte pluridisciplinaire HAL, est destinée au dépôt et à la diffusion de documents scientifiques de niveau recherche, publiés ou non, émanant des établissements d'enseignement et de recherche français ou étrangers, des laboratoires publics ou privés. 


\title{
Vibration-induced compaction of granular suspensions
}

\author{
S. Kiesgen de Richter ${ }^{1 \mathrm{a}}$, C. Hanotin ${ }^{1}$, N. Louvet ${ }^{1}$, S. Leclerc ${ }^{1}$, F. Demeurie ${ }^{1}$, and P. Marchal ${ }^{2}$ \\ 1 Laboratoire d'Energétique et de Mécanique Théorique et Appliquée (LEMTA), Université de Lorraine-CNRS, UMR 7563, \\ Vandoeuvre-lès-Nancy, F-54504, France \\ ${ }^{2}$ Laboratoire Réaction et Génie des procédé (LRGP), Université de Lorraine-CNRS, UMR 7274, Nancy, F-54001, France
}

Received: date / Revised version: date

\begin{abstract}
We study the compaction dynamics of vibrated granular suspensions using a digital imaging technique and MRI measurements. Starting from initialy loose packings and after an initial transient behavior, the dynamics exhibits two regimes: a fast regime at short times where the packing fraction increases linearly and a slow logarithmic regime at larger times. The transition time between these two regimes, $\tau_{c}$, depends in particular on the fluid viscosity, vibrations intensity and grains diameter. We show that $\tau_{c}$ increases linearly with the lubrication Peclet number, $P e_{L u b}$ introduced by Hanotin et al. [1] to study the steady-state rheological behavior of both sheared and vibrated granular suspensions. Furthermore, we perform MRI measurements to study the spatial evolution of the compacity in the depth of the vibrated packing during compaction. A rising compaction front whose velocity decreases with $P e_{L u b}$ propagates in the packing. Dilatancy and memory effects are not observed when the vibration intensity is instantaneously increased. Irreversible behavior is yet evidenced when the vibration intensity is instantaneously reduced. A phenomenological two states model, based on free volume arguments and cooperative rearrangements explains both the existence of the two regimes and the properties of the compaction front velocity.
\end{abstract}

PACS. PACS-key discribing text of that key - PACS-key discribing text of that key

\section{Introduction}

Granular dispersions are dense systems made of spherical or arbitrarily shaped particles of size higher than a few hundred micrometers that are completely or partially filled with an interstitial fluid. Contrary to colloids, granular dispersions are non-Brownian. This feature allows peculiar flow properties (jamming, localization, aging) which are not well understood yet. Granular dispersions are widely found in industrial processes as diverse as food manufacturing (cereals), pharmaceutical manufactures (powders, medicines), construction industry (sand, concret), paper industry (fibers, paper pastes) or mining engineering (clay or ores) for example. Some commercially important issues concern the optimization of their transport, storage or mixing properties. The packing fraction of those granular materials controls their rheological behavior and becomes therefore a relevant parameter for a broad range of applications to reduce the costs for their manipulation. The compaction process of dry granular materials has extensively been studied in the litterature [2-10]. Some of these studies $[2,3]$ have experimentally shown that the compaction of dry granular systems presents a slow increase of the packing fraction when series of taps are applied. Among others, two laws are proposed: an inverse logarithmic law with the tapping number[2]

$$
\phi(\infty)-\phi(t) \propto \frac{1}{\log (t)}
$$

or a stretched exponential law [3]

$$
\phi(\infty)-\phi(t) \propto \exp \left(-\left(\frac{t}{\tau}\right)\right)^{\beta}
$$

with a characteristic compaction time $\tau$ which depends on the tap amplitude. Althought compaction dynamics has extensively been studied for dry granular systems, the compaction of fluid saturated granular suspensions is still open. To our knowledge, only one study performed by Lesaffre et al. [11] concerns the compaction of viscous fluid saturated granular suspensions subjected to discrete taps. Authors show that the compaction is slower than in dry packings and follows a logarithmic law (equation 1) but an open question remains as regards how external parameters such as fluid viscosity, grain diameter or tap intensity influences the compaction dynamics. In this work, we focus on the compaction dynamics of viscous fluid saturated granular suspensions not subjected to individual taps but sinusoidal vibrations of given amplitude and frequency. Vibrations are sufficiently low so that grains keep contact with their neighbour. We use digital imaging technique to probe the global compacity of the 
packing, $\phi(t)$ and MRI imaging techniques to study local inhomogeneities[12-14] during compaction. We experimentally observe two regimes: a fast linear regime at short times precedes a slow logarithmic regime. The transition between the two regimes is controlled by the lubrication Peclet number, $P e_{l u b}$ previously introduce by Hanotin et al. $[15,1]$ to quantify the rheology of vibrated suspensions. In particular, no stationary state is reached in the accessible experimental time and the compacity at a given delay time increases with the energy injected by vibrations. MRI measurements show that a compaction front propagates from the bottom to the top of the packing with a velocity which decreases with $P e_{l u b}$. Our results also show that contrary to dry granular systems, no dilatancy effect is observed in fluid saturated granular suspensions subjected to low amplitude sinusoidal vibrations. The absence of observable stationary state and the front propagation velocity is well accounted by a phenomenological two state model related to the inherent bimodal behavior of chain forces in granular packing.

\section{Experimental methods}

\subsection{Materials preparation}

We study the compaction dynamics of fluid saturated monodisperse granular suspensions made of spherical glass or zirconium beads ${ }^{1}\left(\rho_{\text {glass }}=2200 \mathrm{Kg} \cdot \mathrm{m}^{-3}\right.$ and $\rho_{\text {zirco }}=$ $\left.6600 \mathrm{Kg} \cdot \mathrm{m}^{-3}\right)$ using digital imaging and MRI measurements. Three bead diameters are used $(100 \pm 5 \mu \mathrm{m}, 335 \pm$ $15 \mu \mathrm{m}, 530 \pm 30 \mu \mathrm{m})$ with different fluid viscosities, $\eta_{f}$ ranging from $10^{-3}$ to $10^{-1} \mathrm{~Pa}$.s. The sample is pourred into a cylindrical plexiglass vessel $(20 \mathrm{~mm}$ Diameter, $10 \mathrm{~cm}$ height) and sinusoidal vertical vibrations are applied via a shaker. The amplitude $[20-200 \mu \mathrm{m}]$ and the frequency $[30-70 \mathrm{~Hz}]$ of vibrations are controlled by a closed loop system (Dactron comet USB) connected to a power amplifier and an accelerometer attached to the vessel (figure 1). Before each experiment starts, the glass tube is filled with the interstitial fluid (Water-Emkarox ${ }^{2}$ mixture) of given viscosity. Then, grains are poured little by little in the tube with a funnel. With this method, grains falls slowly forming a loose packing with an initial packing fraction, $\phi_{0}$ of order $0.58 \pm 0.01$. We verify that all grains have sedimented before each experiment starts. The time one needs to obtain the initial state can be more or less depending on the interstitial fluid viscosity. This procedure gives a reproducible initial state with a flat surface at the top of the packing. In next sections, we present results on the evolution of the packing fraction $\phi(t)$ by applying well controled sinusoidal vibrations of given amplitude, $A$ and frequency, $f$. Experiments are performed for different values of the $\Gamma$ number defined as the ratio of the vibrational

\footnotetext{
1 Zirconium beads are used for MRI experiments. Their density being larger than the density of glass beads, the compacity variation is therefore larger and compatible with the MRI resolution.

${ }^{2}$ water soluble polyalkylene glycol
}

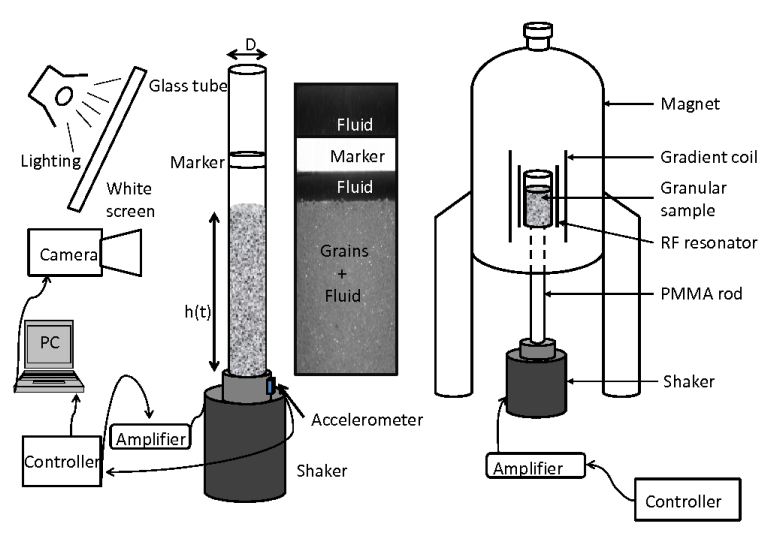

Fig. 1. Sketch of the two exploited experimental set-up.(Left) Digital imaging experimental set-up. The granular suspension is placed in a vertical glass tube subjected to sinusoidal vibrations of given frequency and amplitude. The camera is triggered for strobed image acquisition. An image is taken every five vibration period. (Right) Sketch of the MRI experimental set-up. Vibrations are transmitted from the shaker to the granular sample placed in the spectrometer via a PMMA rod.

peak acceleration over the gravitationnal acceleration $g$, $\Gamma=A \omega^{2} / g$ where $\omega=2 \pi f$ is the angular frequency. In our experiments, the values of $\Gamma$ ranges from 1 to 5 .

\subsection{Digital imaging measurements}

The compacity is obtained from the height $h(t)$ of the granular column measured with a camera. A ring-shaped white marker, whose height from the bottom of the tube is known, is attached to the tube to calibrate the measurement of $h(t)$. The camera is triggered for strobed image acquisition, such that the camera takes an image every 5 vibration periods. The tube is lighted up from the front with a lamp and a white screen which produce an homogeneous diffuse light. The position of the granular/fluid interface is measured by image analysis via an algorithm especially developed for this task. The variation of pixel values along the vertical is analysed.

An abrupt variation at the interface grains/fluid is detected so that the maximum of the first derivative of the pixel values variations gives the position of the interface. The compacity, $\phi(t)$ is then obtained through the value of $h(t)$, the density of grains, $\rho$, the diameter of the vessel, $D$ and the weigth of the entire column of grains, $m$, with $\phi(t)=m / \rho \pi R^{2} h(t)$. Our method gives an accurate measure of $\phi(t)$ variations with a high resolution. The accuracy on $h(t)$ is typically limited by the pixel size of the camera $(\approx 10 \mu \mathrm{m})$ which gives an error of about $0.02 \%$ in $\phi(t)$. However, this method gives a relatively poor accuracy in the absolute value of $\phi(t)$ due to image calibration, density of the beads and pixel intensity averaging. We estimate that the systematic error on the packing fraction measurement is about $2 \%$. 


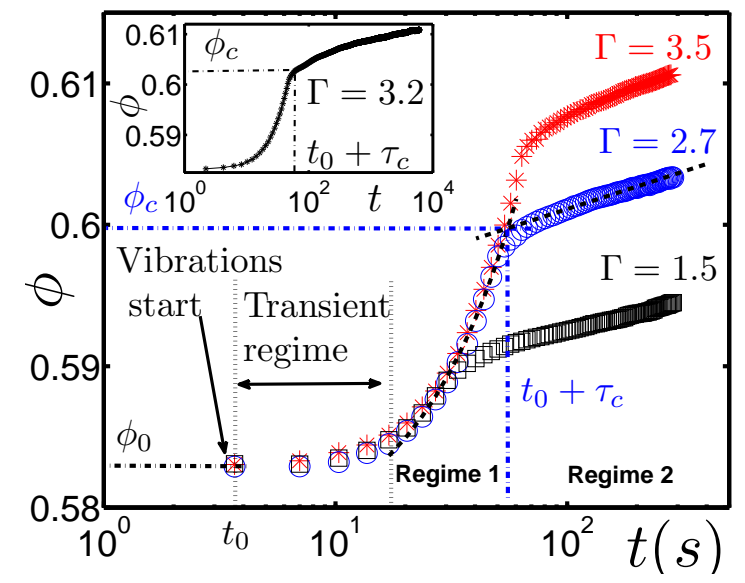

Fig. 2. Packing fraction $\phi(\mathrm{t})$ as a function of time $t$ for glass beads $(335 \mu \mathrm{m})$ immersed in a water-emkarox mixture $\left(\eta_{f}=19 \mathrm{mPa} . \mathrm{s}\right)$ obtained by digital imaging measurements. Three different cases are illustrated: $\Gamma=$ 1.5(squares), 2.7(circles) and 3.5(crosses). The dash lines are fitted in the linear $(\Phi \sim t)$ and logarithmic $(\Phi \sim \log (t))$ regime respectively after a short transient regime. Measurements have been performed at the same vibrations frequency $f=30 \mathrm{~Hz}$. Inset: Experimental compaction curve at long time $(\Gamma=3.2)$ showing that no stationary state is reached even after 2 Hours.

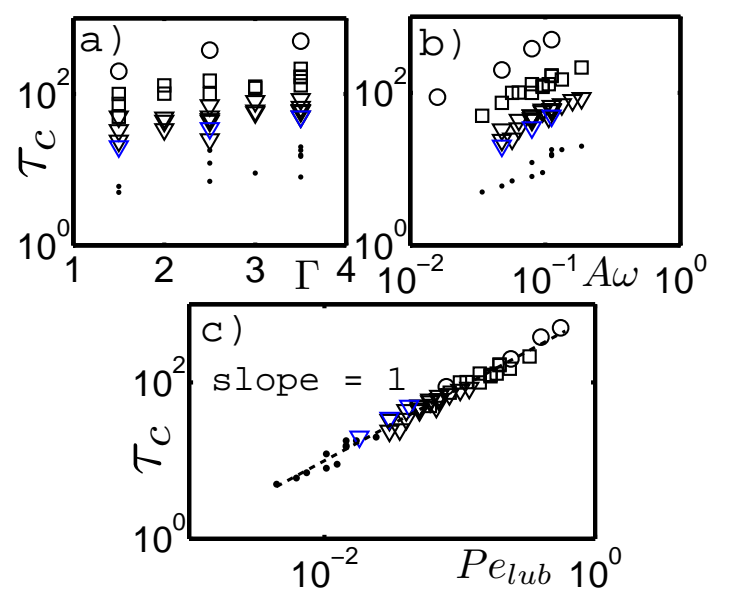

Fig. 3. Critical compaction time $\tau_{c}$ as a function of $\Gamma(\mathrm{a}), A \omega(\mathrm{b})$, and the lubrication Peclet number $P e_{l u b}=$ $\eta_{f} 2 \pi A f / P D$ (c). Experiments were carried out for $335 \mu \mathrm{m}$ and $500 \mu \mathrm{m}$ glass beads immersed in water-emkarox mixtures of different viscosities $\eta_{f}$ and vibration intensities $(A, f)$. $\eta_{f}=3.9 \mathrm{mPa} . \mathrm{s}, \mathrm{D}=300 \mu \mathrm{m} ; \nabla \eta_{f}=19 \mathrm{mPa} . \mathrm{s}, \mathrm{D}=300 \mu \mathrm{m} ; \square$ $\eta_{f}=53 \mathrm{mPa} . \mathrm{s}, \mathrm{D}=300 \mu \mathrm{m} ; \bigcirc \eta_{f}=150 \mathrm{mPa} . \mathrm{s}, \mathrm{D}=300 \mu \mathrm{m}$ $\diamond \eta_{f}=19 \mathrm{mPa}$.s, $D=500 \mu \mathrm{m}$

\subsection{Magnetic resonance imaging measurements}

Magnetic Resonance Imaging measurements give us information on the local packing fraction along the depth of the suspensions during compaction. We acquire 1D profiles along the height of the packing instead of the typical $2 \mathrm{D}$ images to reduce data acquisition time. This method allows us to achieve a time resolved monitoring of the compaction dynamics. In this case, the intensity of each pixel is proportional to the proton density in the liquid at a given depth. Another advantage of the 1D experiment is that one avoids vibration artifacts arising from a phase encoding procedure. The experiments were carried on a Bruker Avance III $600 \mathrm{MHz}$ spectrometer equipped with a MicWB40 gradient coil and a $2.5 \mathrm{~cm}$ diameter quadrature resonator. The granular sample is placed in a special container that was designed to adapt to the specification of this probe, while allowing easy manipulation and a good control of the vibrations. Images were acquired using a classical spin-echo experiment with the following parameters: repetition time $(T R): 5 \mathrm{~s}$, echo time $(T E): 6.9 \mathrm{~ms}$, field of view $(F O V): 5 \mathrm{~cm}$, matrix : 512 points, spatial resolution $98 \mu \mathrm{m}$. The repetition time $T R$ was chosen sufficiently long to avoid longitudinal relaxation contrast. $T E$ was chosen as short as possible, but there is nevertheless a decrease in the signal due to the short transverse relaxation time. In order to take in account this effect in the data processing, T2 was measured using a classical Carr Purcell Meiboon Gill method on compacted and non-compacted samples. This relaxation time did not significantly change between the two states. The measured profile was also corrected to take into account the non homogeneous radiofrequency field profile. The packing fraction at each pixel is calculated from the ratio of the corrected intensity of the signal $I$ over the reference intensity $I_{\text {ref }}$ measured when the container is only filled with fluid.

\section{Evolution of the compacity}

\subsection{Influence of the vibrations parameters and the interstitial fluid viscosity on the compaction}

We analysed the compaction dynamics for different values of $\Gamma$ and intersitial fluid viscosity $\eta_{f}$. Figure 2 shows the evolution of the compacity obtained by digital imaging measurements as a function of time for 3 different values of $\Gamma(1.5 ; 2.7 ; 3.5)$ for $\eta_{f}=19 \mathrm{mPa} . \mathrm{s}$ and $f=30 \mathrm{~Hz}$.

The compaction dynamics depends on $\Gamma$ and two regimes are evidenced after a short transient period. The transient regime lasts few seconds and comes from the sarting up of vibrations. Then, a fast dynamics at short time scale (regime 1) is observed and the dynamics slows down after a critial time $\tau_{c}$ corresponding to a compacity $\Phi_{c}$ (regime 2 ). The existence of two distinct regimes shows that laws 1 and 2 can not describe the entire compaction dynamics of granular suspensions. The evolution of the whole compacity in the first regime does not depend on $\Gamma$ and is linear $(\Phi(t) \propto t)$. We see that all curves collapse on the same master curve in this regime. After a critical time $\tau_{c}$ which increases with $\Gamma$ and related to a critical compacity $\Phi_{c}{ }^{3}$, the system reaches the regime 2. A slowing down of the compaction process is then observed with a logaritmic increase of the compacity with time $(\Phi(t) \propto \log (t))$. As shown in the inset of figure 2, the compacity doesn't reach a stationary state in our experimental window of

\footnotetext{
$3\left(\Phi_{c}, \tau_{c}\right)$ are experimentaly extracted from the fits as the intersection between the linear and the logarithmic regime
} 


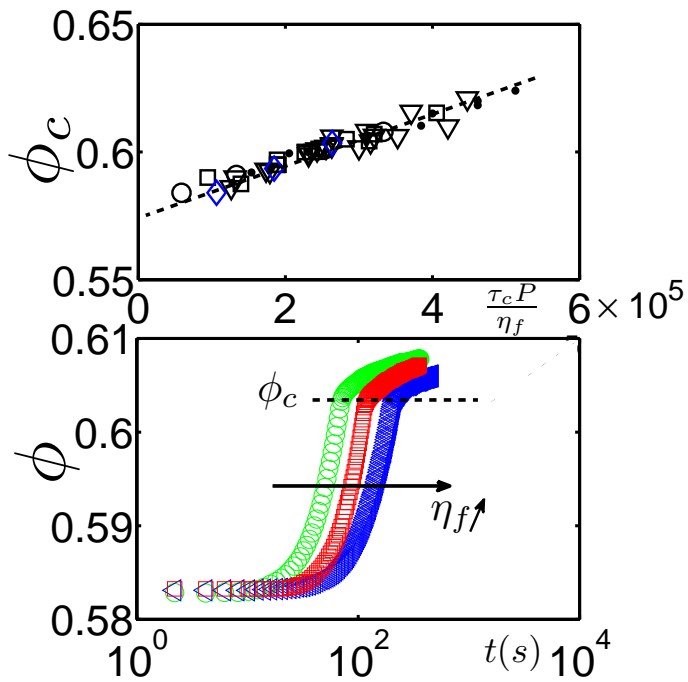

Fig. 4. (top) Critical packing fraction $\phi_{c}$ as a function of $\tau_{c} P / \eta_{f}$. Experiments were carried out for $335 \mu \mathrm{m}$ and $500 \mu \mathrm{m}$ glass beads immersed in water-emkarox mixtures of different viscosities $\eta_{f}$ and vibration intensities $(A, f)$. $\bullet \eta_{f}=$ $3.9 \mathrm{mPa}$.s,$D=300 \mu \mathrm{m} ; \nabla \eta_{f}=19 \mathrm{mPa} . \mathrm{s}, D=300 \mu \mathrm{m} ; \square$ $\eta_{f}=53 \mathrm{mPa} . \mathrm{s}, D=300 \mu \mathrm{m} ; \bigcirc \eta_{f}=150 \mathrm{mPa} . \mathrm{s}, \mathrm{D}=300 \mu \mathrm{m}$; $\diamond \eta_{f}=19 \mathrm{mPa}$.s, $D=500 \mu \mathrm{m}$ (bottom) Evolution of the compacity $\Phi$ with time for three different viscosities $\left(\bigcirc \eta_{f}=\right.$ $19 \mathrm{mPa}$ P.s, $\left.\square \eta_{f}=35 \mathrm{mPa} . \mathrm{s}, \triangleleft \eta_{f}=60 \mathrm{mPa.s}\right)$. Experiments were performed for $335 \mu \mathrm{m}$ beads diameter at the same vibrational intensity $(\Gamma=3.4, f=50 \mathrm{~Hz})$ and show that $\phi_{c}$ does not depend on $\eta_{f}$.

time. In the last regime, the system shows a "glassy" behavior and ages slowly. This result shows that, starting from a loose packing, no stationary state can be reached in usual rheological experiments where sinusoidale small amplitude vibrations are applied.

In the following, we focus on the transition point $\left(\tau_{c}, \phi_{c}\right)$ and its dependency with : fluid viscosity, vibration intensity and grain diameters. The figure $3 \mathrm{a}$ ) and b) show the critical time $\tau_{c}$ as a function of the $\Gamma$ parameter and the vibration liftoff velocity $A \omega$ for different fluid viscosities $\eta_{f}$ and vibration properties $(A, f)$. From these data, neither $\Gamma$ nor $A \omega$ are the appropriate parameter that controls $\tau_{c}$ for sinusoidal vibrations as usually assumed in dry granular packings [6]. In particular, $\tau_{c}$ highly depends on $\eta_{f}$ as shown in figure $3 \mathrm{~b}$ ). To interprete the results of figure 3 , We assume in the following that the compaction mechanism of granular suspensions is governed by the competition between the granular pressure of the packing $P=\Delta \rho g \phi(t) h(t)$, where $\Delta \rho$ is the relative density of beads and fluid, $g$ the gravity and the lubrication stress, $\sigma_{l u b} \propto \eta_{f} A 2 \pi f / D$ induced by vibrations which provides more degrees of freedom to the particles to rearrange (see [1]). Figure $3 \mathrm{c}$ presents $\tau_{c}$ as a function of the lubrication Peclet number $P e_{l u b}=\sigma_{l u b} / P=2 \pi \eta_{f} A f / P D$. All points fall onto a master curve with a slope +1 , extending over two decades in both $P e_{l u b}$ and $\tau_{c}$, which validates the proposed approach. We conclude that the $P e_{l u b}$ number is the appropriate control parameter to describe the tran-

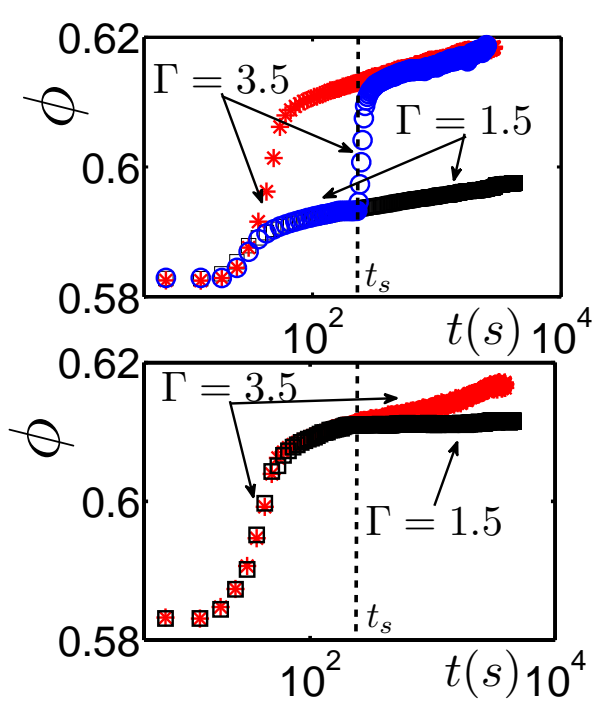

Fig. 5. (top) Evolution of the compacity $\Phi$ after the $\Gamma$ number is switched from 1.5 to 3.5 (blue circle) at $t=t_{c}$. The reference compaction curves for $\Gamma=1.5$ (black square) and $\Gamma=3.5$ (red stars) are plotted. The packing fraction suddently increase when $\Gamma$ is switched. (bottom) Evolution of the compacity $\Phi$ after the $\Gamma$ number is switched from 3.5 to 1.5 (black square) at $t=t_{c}$. The reference compaction curve for $\Gamma=3.5$ is plotted. The packing fraction stops to increase when $\Gamma$ is switched down and an effective stationary state is reached where $\Phi$ is constant.

sition between the linear and the logarithmic regime. The transition between the two regimes occurs at a critical compacity $\phi_{c}$ related to $\tau_{c}$ and $P e_{l u b}$.

Figures 4 show that $\phi_{c}$ not only depends on $\tau_{c}$ but also on the interstial fluid viscosity $\eta_{f}$ as $\phi_{c} \propto \tau_{c} P / \eta_{f}$. For a given $A f$, we find $\tau_{c} \propto P e_{l u b} \propto \eta_{f}$ showing that $\phi_{c}$ does not depend on $\eta_{f}$. It comes that $\phi_{c} \propto A f / D$ and that the critical time $\tau_{c}$ can be interpreted as the typical time a particle takes to move over a distance equals to its diameter in a fluid of effective viscoty $\eta_{e f f}=\eta_{f} \phi_{c}$ where the critical packing fraction $\phi_{c}$ depends on grain diameter and vibrational velocity. $\phi_{c}$ is then a critical packing fraction above which cooperative effects appear. As long as the packing is loose, interstitial voids are accesible to individual grains movement, and densification is fast (regime $1)$. When the packing fraction reaches the critical value $\phi_{c}$ the filling of voids requires the cooperation of many grains and becomes slower (regime 2).

\subsection{Memory effects and dilatancy}

In this section, we investigate the evolution of the compacity when the vibrational intensity is suddently switched. Two cases are considered according to the vibrational energy increases or decreases. Figure 5 (up) shows the behavior of the compacity when the $\Gamma$ number suddenly increases from 1.5 to 3.5 at $t=t_{s}$. The compacity increases at $t_{s}$ and reached the reference curve $\Gamma=3.5$. This behavior shows that the compacity of the system does not depend on its history when the vibrational intensity increases. No memory effects and dilatancy are observed in 


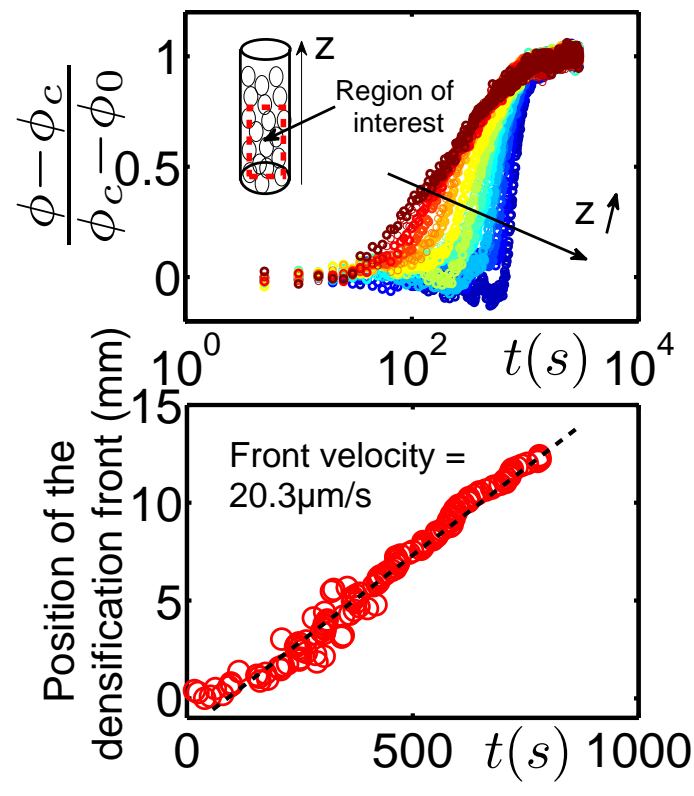

Fig. 6. (top) Evolution of the normalized compacity $(\phi-$ $\left.\phi_{c}\right) /\left(\phi_{c}-\phi_{0}\right)$ as a function of time for different depth, $z$, in the packing. Data are obtained in the region of interest where MRI measurements are performed for $\eta_{f}=560 \mathrm{mPa} . \mathrm{s}, \mathrm{D}=100 \mu \mathrm{m}$, $\Gamma=2$ and $f=80 \mathrm{~Hz}$. (bottom) Position of the compaction front as a function of time in the region of interest extracted from the evolution of the scaled compacity. The front is defined as the inflexion point (maximum of the first derivative) of the compaction curve

this case. An increase of the tap amplitude immediately compactes the packing to a new compacity related to the new amplitude. On the contrary, the figure 5 (bottom) shows the inverse case when $\Gamma$ suddenly decreases from $\Gamma=3.5$ to $\Gamma=1.5$ at $t=t_{s}$. In this case, the compacity suddenly stops to increase and an effective stationary state is reached when the vibrational intensity is reduced. This propertie can be used to prepare a packing with a well controlled compacity at initial time to perform experiments in stationary conditions $[1,15]$. For that, large amplitude vibrations are applied to the packing during a given time duration and measurements are performed at lower amplitudes. With that experimental protocol, experiments can be conducted at imposed packing fraction under vibrations. In addition, when large intensity vibrations are applied, the system reaches a dense state rapidly whith large local cohesion stresses and then when vibrations are reduced, the injected energy by vibrations is not sufficient to cause large rearrangements and the compaction dynamics stops.

\section{Inhomogeneities in the depth of the packing during granular compaction}

We describe below experimental results concerning MRI measurements used to study the inhomogeneity of $\phi$ in the depth of the packing during compaction. The experimental protocol and methods are described in part 2. MRI

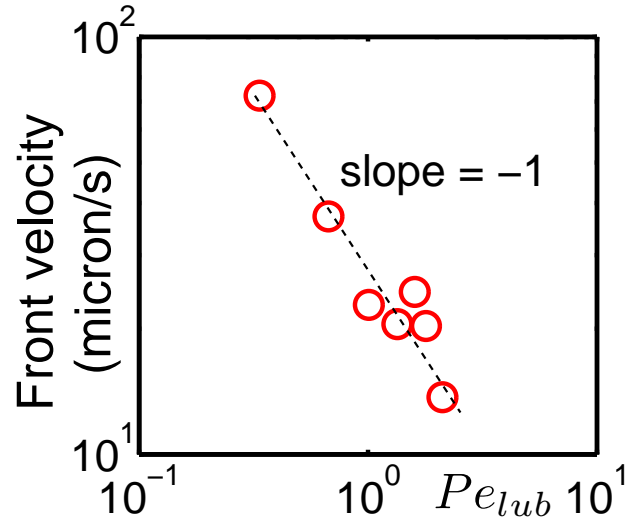

Fig. 7. Evolution of the front velocity extracted from the position of the densification front as a function of the lubrication peclet number, $P e_{l u b}$. The slope -1 is a guide for eyes.

technique allows us to monitore the evolution of the local compacity as a function of time in a region of interest close to the bottom of the packing. Even though the precision on the absolute value of the compacity is poor, the technique gives us an accurate method to monitore the variations of the compacity as a function of time at a given depth.

Figure 6 top shows the evolution of the normalized packing fraction $\left(\phi-\phi_{c}\right) /\left(\phi_{c}-\phi_{0}\right)$ with time for different values of the depth, $z$ along the height of the packing. At a given depth, the evolution is similar to the one obtained by macroscopical measurements (see figure 2). A fast linear regime appears at short times and is followed by a slow log regime. Compaction curves shows that the compacity highly depends on the depth, $z$. The compaction process begins at the bottom of the vessel and a compaction front propagates slowly toward the top of the packing . It propagates linearly with time with a typical constant velocity of the order of tens micrometers per seconds (fig 6 bottom). This result suggests that the system compacts by succesive layers. The velocity of the front depends on the experimental parameters previously introduced. This dependency is not trivial since the propagation velocity depends on the critical packing fraction $\phi_{c}$. For larger $\phi_{c}$, a given layer would take more time to compact and the front velocity would decrease. Figure 7 shows the evolution of the front velocity as a function of the previously introduced $P e_{l u b}$ number. The velocity decreases in log$\log$ with $P e_{l u b}$ with a slope closed to $-1^{4}$. It decreases with the interstitial fluid viscosity $\eta_{f}$ and with the energy injected by vibrations as the typical compaction time $\tau_{c}$ previously introduced. When $P e_{l u b}$ increases, $\tau_{c}$ increases and a given layer takes more time to compact. The front velocity thus decreases.

4 The low precision on the value of the front velocity which depends on the heterogeneities in the packing could explain the discrepancy. 


\section{Interpretation: a phenomenological two state model}

\subsection{Free volume arguments}

In this section, we propose a simple phenomenological model based on free volume arguments to describe the compaction dynamics of vibrated granular suspensions. The model describes the compaction dynamics as a transition between two states: a "mobile state" (M) where particles are free to rearrange (largest free volumes per particles) and a "consolidated state" (C) where particles are jammed (critical free volume per particle (see figure 8 left and [16]). The variable of state used is the fraction of grains in the state $(\mathrm{C}), P_{c}$, defined as $P_{C}=N_{C} / N_{P}$ with $N_{C}$ the number of grains in the state $C, N_{P}$ the total number of grains and $P_{M}$ the occupation probability of state (M) such as $P_{M}+P_{C}=1$. The temporal evolution $\dot{P}_{C}$ is given by:

$$
\begin{aligned}
\dot{P}_{C}(t) & =\omega_{C M}(t) P_{M}(t)-\omega_{M C}(t) P_{C}(t) \\
= & \left(1-P_{C}(t)\right) \omega_{C M}(t)-\omega_{M C}(t) P_{C}(t)
\end{aligned}
$$

where $\omega_{C M}$ and $\omega_{M C}$ are probabilities of transition per unit time between states $(\mathrm{M})$ and $(\mathrm{C})$. To model compaction, we suppose that particles can not escape from the state $(\mathrm{C})\left(\omega_{M C}=0\right)$ but can escape from the state $(\mathrm{M})$ depending on the free volume and the energy injected by vibrations. Assuming a Maxwell-Boltzmann distribution of free volumes in vibrated granular matter[15,20-22], we suggest that transitions are activated by vibrations and occur at a rate $\omega_{C M}(t) \propto f_{0} \exp \left(-\frac{\xi v_{f}^{*}}{\overline{v_{f}}}\right)[15,17-19]$ where $\overline{v_{f}}$ is the mean free volume per particles, $v_{f}^{*}$ a characteristic free volume beyond which spatial rearrangements of neighbouring contacts become possible, $\xi$ is an overlap factor of the grains free volumes and $f_{0}$ a typical grains reorganization frequency. $\bar{v}_{f}$ is related to the compacity, $\overline{v_{f}}=v_{g}\left(1 / \phi(t)-1 / \phi_{R C P}\right)$ where $v_{g}$ is the volume of a single particle and $\phi_{R C P}$ is the random close-packing limit $(\approx 0.64)$. Assuming that ${ }^{5} v_{f}^{*}=K v_{g}$ where $K$ is a constant,

$$
\omega_{C M}(t) \propto f_{0} \exp \left(-\frac{K_{1}}{\frac{1}{\phi(t)}-\frac{1}{\phi_{R C P}}}\right)
$$

with $K_{1}=K \xi$.

To allow experimental assessment, we can reasonably assume that ${ }^{6} \phi(t) \sim P_{C}(t)$. The kinetic equation 3 is transformed into a differential constitutive equation on $\phi(t)$.

$$
\dot{\phi}(t)=(1-\phi(t)) f_{0} \exp \left(-\frac{K_{1}}{\frac{1}{\phi(t)}-\frac{1}{\phi_{R C P}}}\right)
$$

\footnotetext{
${ }^{5} v_{f}^{*}$ scales with grain diameter and is purely geometric as $K$. It should not depend on vibrations properties contrary to $\xi$

${ }^{6}$ Starting from loose packings, the packing fraction increases with $P_{c}(t)$
}

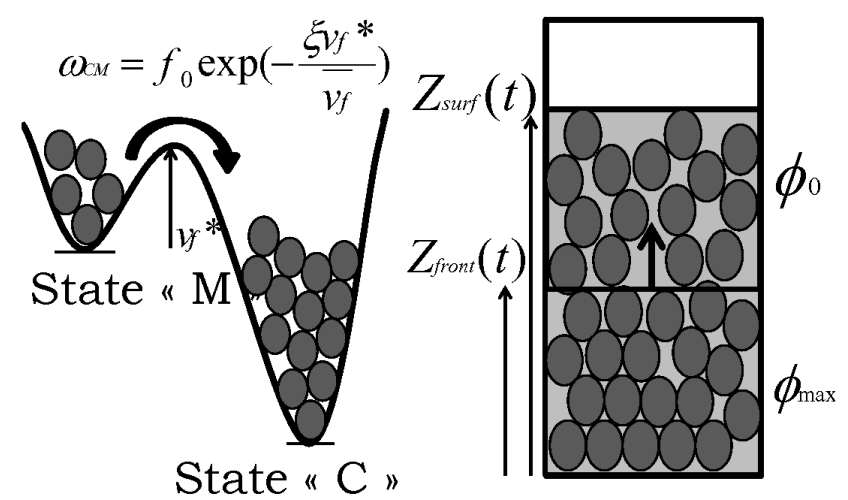

Fig. 8. (Left) Sketch of the phenomenological 2 state model. Grains can move from a loose state $M$ to a consolidated state $C$ depending on the transition rates $\omega_{C M}$ and $\omega_{M C}$. (Right) Sketch of the compaction front propagation. $Z_{\text {front }}(t)$ is the position of the interface which separates the packing in two states: a consolidated state at $\phi_{\max }$ and a loose state at $\phi_{0}$.

We plot in figure 9, the evolution of $\phi(t)$ as a function of $t$ for different values of the $K_{1}$ parameter. As observed experimentally, a slow regime appears when the compacity gets close to the critical packing fraction $\phi_{R C P}$. At the opposite, for $\phi<<\phi_{R C P}$ (short times)there is a fast variation of the compacity. The transition time between the two regimes increases with the $K_{1}$ parameters and the typical reorganisation frequency $f_{0}$. The slow regime observed at large times comes from the existence of the critical free volume $v_{f}^{*}$. When the mean free volume per particles becomes of the order of $v_{f}^{*}$, the probability for a grain to fall in a hole formed by its neighbour decreases and the compaction dynamics slow down. The compacity evolves slowly and evidences an "ageing" phase. Compacity curves are shifted when $f_{0}$ decreases as observed experimentally when the interstitial fluid viscosity increases (see figure 4) This suggest that $f_{0}$ is proportional to the inverse of the time a particle needs to move through the fluid of viscosity $\eta_{f}$ to enter into direct contact with another particle under the granular pressure $P, f_{0} \propto P / \eta_{f}$. This predicts that the typical compaction time should increase linearly with $\eta_{f}$ as observed experimentally. The evolution of the compaction curves whith $K_{1}$ also suggests that $K 1$ decreases when the vibration energy increases as $f_{0}$. Despite the fact that our approach remains at a mean-field level, it is able to explain the existence of a fast regime at short times where grains rearrange easily and a slow regime at larger times where a gradual ageing of the packing is observed as a result of the existence of a critical free volume beyond which spatial rearrangements of neighbouring contacts become possible.

\subsection{Compaction front propagation}

MRI measurements highlights the existence of a compacity front in the depth of the packing whose velocity de- 


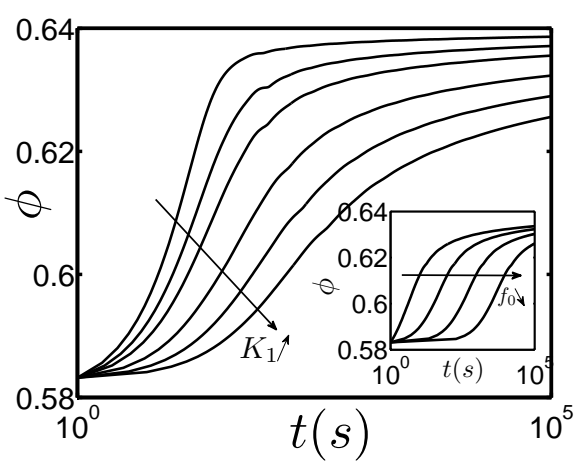

Fig. 9. Compacity as a function of time given by equation 5 for different values of $K_{1}$ parameter with $f_{0}=10^{-2}, \phi_{R C P}=0.64$. From top to bottom, le lines corresponds to $K_{1}=0.05,0.1$, $0.15,0.25,0.35$ and 0.5 respectively. Inset: The corresponding results for $K_{1}=0.25$ and $\Phi_{R C P}=0.64$ with $f_{0}=10^{-1}, 10^{-2}$, $10^{-3}$ and $10^{-4}$.

creases with $P e_{l u b}$ in the linear regime. To interprete this result, we suppose that the transition from the state $\mathrm{M}$ to the state $\mathrm{C}$ is inhomogeneous in the depth of the packing and begins at the bottom of the vessel. The packing can then be separated in two parts: a bottom part where the compacity, $\phi(t)$ equals the final compacity $\phi_{\max }{ }^{7}$ and an upper part where the packing fraction keeps equal to the initial packing fraction $\phi_{0}$ (see figure 8 right). The bottom part has a height $Z_{\text {front }}(t)$ and the the position of the free surface of the packing is $Z_{\text {surf }}(t)$. Mass conservation during the compaction process gives:

$$
Z_{\text {surf }}(0) \phi_{0}=Z_{\text {front }}(t) \phi_{\max }+\phi_{0}\left(Z_{\text {surf }}(t)-Z_{\text {front }}(t)\right)
$$

Assuming a linear evolution of the height of the free surface at short times (linear regime, see section 3$), Z_{\text {surf }}(t)=$ $Z_{\text {surf }}(0)-\alpha t$ where $\alpha$ is constant during the compaction process. The velocity of the front $\dot{Z}_{\text {front }}(t)$ writes:

$$
\dot{Z}_{\text {front }}(t)=\frac{\phi_{0} \alpha}{\phi_{\max }-\phi_{0}}
$$

According to section 3, $\alpha$ depends on the slope of the compacity in the linear regime and is proportional to $P / \eta_{f}$. In the same way, $\phi_{\max }$ is proportional to the critical compacity $\phi_{c}$ and scales with $A f / D$. At low packing fractions, Equation 7 predicts then a constant $\dot{Z}_{\text {front }}$ proportional to $\phi_{0} \alpha / \phi_{\max } \propto \eta_{f} A f / P D=P e_{l u b}^{-1}$ as observed experimentally (see figure 7 ).

\section{Conclusion}

In this article, we evidence the existence of two regimes during compaction of gravitational suspensions. A linear regime appears at short times whose slope increases with

\footnotetext{
${ }^{7} \phi_{\max }$ is the final packing fraction reached for a given fluid viscosity, grain diameter and vibration intensity.
}

the insterstitial fluid viscosity. We do not observe a stationary state in the experimental window time. The linear regime comes before a second regime whose compacity evolves slowly (log regime). The second regime can last many hours. The transition between these two regimes occurs at a critical packing fraction, $\phi_{c}$ which increases with $\frac{A f}{D}$ related to a critical time $\tau_{c}$ which scales with the lubrication Peclet number $P e_{l u b}^{-1}$. Experiments where vibration intensity is suddenly changed show that no dilatancy is observed when the vibration increase but compaction dynamics speed up. MRI measurements highlight the existence of a compaction front that propagates in the height of the packing during compaction. Its velocity is constant and scales with $P e_{l u b}^{-1}$. A phenomenological two state model which takes into account the inhomogeneity in the depth of the packing predicts the scalings observed experimentally. Free volume arguments explain the slowing down of the dynamics in the second regime when the mean free volume per particles approaches the criticical free volume beyond which spatial rearrangements are possible.

\section{References}

1. C. Hanotin, S. K. de Richter, P. Marchal, L. J. Michot, and C. Baravian, Phys. Rev. Lett, 108, (2012) 198301.

2. J. Knight, C. Fandrich, C. N. Lau, H. Jaeger and S. Nagel, Phys. Rev. E 51,(1995) 3957-3963

3. P. Richard, M. Nicodemi, R. Delannay, P. Ribire and D. Bideau, Nature Materials 4 (2005) 121-128

4. G.Lumay and N.Vandewalle, PRL, 95 (2005) 028002

5. G.Lumay and N. Vandewalle, Phys.Rev.E, 74 (2006) 021301

6. J. A. Dijksman and M. van Hecke, EPL, 88 (2009)44001

7. N. Mueggenburg, Phys.Rev.E, 85 (2012) 041305

8. S.Zivkovic et al., Eur. Phys. J. B, 86 (2013) 461

9. M.Nicolas, P.Duru, O.Pouliquen, Eur.Phys.J.E, 3 (2000) 309

10. O.Pouliquen, M. Belzons, M. Nicolas, Phys. Rev. Lett. 91 (2001) 014301

11. C. Lesaffre, V. Mineau,D. Picart and H.Van damme, C. R. Acad. Sci. Paris 1,(2000) 647-653

12. E. Fukushima, A.D. Rosato, M. Kos, Rev.Sci.Instrum., 68 (1997) 4217-4220

13. M.D. Mantle, A. Sederman, L.F. Gladden, J.M. Huntley, T.W. Martin, R.D. Wildman, M. D. Shattuckc , Powder Technology, 179 (2008) 164-169

14. K.SAKAIE et al, EPL, 84 (2008) 38001

15. P. Marchal, L. Choplin and N. Sminari, Journal of rheology, 1 (2009) 1

16. P. Marchal, C. Hanotin, L.J Michot and S. Kiesgen de Richter, Phys. Rev. E 88.1, (2013) 012207.

17. S. F. Edwards and R. B. S. Oakeshott, Physica A 157, (1989) 1080.

18. T. Aste and T. D. Matteo, Phys. Rev. E 77, (2008) 021309.

19. P.G de Gennes, Journal of colloid and interface science 226.1, (2000) 1-4

20. E. Caglioti, A. Coniglio, H.J. Herrmann, V. Loreto and M. Nicodemia, Physica A 265, (1999) 311

21. T. Boutreux and P.G. de Gennes, Physica A 244, (1997) 59

22. P.Richard et al., Phys.Rev.E 68, (2003) 020301 